\title{
Epidemiology of Intensive Care Unit Infections and Impact of Infectious Disease Consultants in Managing Resistant Infections
}

\author{
${ }^{1}$ Ravi, K.P., ${ }^{2}$ Suresh Durairajan, ${ }^{1}$ Sankalp Parivar, \\ ${ }^{1}$ Ramesh Venkataraman, ${ }^{2} \mathrm{~V}$. Ramasubramanian and ${ }^{1} \mathrm{~N}$. Ramakrishnan \\ ${ }^{1}$ Department of Critical Care Medicine, \\ ${ }^{2}$ Department of Infectious Diseases, \\ Apollo Hospitals, Greams Road, Chennai, 600006, India
}

Received 2012-06-26, Revised 2013-02-06; Accepted 2013-05-21

\begin{abstract}
Choice of empiric antibiotics in India have generally been guided by western data and guidelines. However, validity and applicability of western guidelines in the Indian setting is not known. The aim of our study was to explore microbial prevalence and resistance patterns in a tertiary care Intensive Care Unit (ICU) in India and to determine whether western guidelines are still valid for use in the Indian setting. We also wanted to evaluate the impact of infectious disease specialist consultation on the appropriateness of initial antibiotic choice and de-escalation practices. Prospective observational study from January 2009 to July 2009, in a setting of 600 bed multispecialty tertiary care hospital. Relevant samples from patients suspected to have infection were cultured and sensitivity testing was performed according to standard procedures. Only the first positive cultures from each patient were used for analysis of the 401 patients admitted to ICU during the study period $25 \%$ had positive cultures. $60 \%$ of the cultures grew Gram negative organisms with $E$. coli, Pseudomonas and Acinetobacter species being the commonest isolated pathogens. Mortality among culture positive patients in the Intensive Care Unit (ICU) was 31\%. Culture and sensitivity patterns of organisms in Indian ICUs differ from that in the west. Gram negative organisms are most commonly cultured. Initial antibiotic choice when made using western guidelines seem to be appropriate only in (48.4\%) of patients. When choosing empiric antibiotics in acutely ill Indian ICU patients, modifications to western guidelines need to be done using local microbial prevalence and resistance patterns.
\end{abstract}

Keywords: Infection, Intensive Care Unit, Resistance, Infectious Disease Specialist

\section{INTRODUCTION}

Infections in the Intensive Care Units (ICUs) result in increased morbidity, mortality, length of ICU and hospital stay (Weinstein, 1998). Furthermore ICUs act as epicenters for Hospital Acquired Infections (HAI) and the development of Multidrug-Resistant Organisms (MDROs) due to prolonged hospitalization, serious illnesses, compromised hosts and the higher rates and longer duration of antibiotic usage (Kollef and Fraser, 2001). Early and appropriate empiric antibiotic use improves survival in critically ill patients, especially with shock. While routinely choosing broader antibiotics may increase the appropriateness of antibiotic choice, it may induce further resistance and lead to extreme drug resistance. Hence the clinician has to choose empiric antibiotics aiming to both maximizing outcomes and minimizing emergence of resistance. Due to lack of local data from India, western guidelines on initial antibiotic selection are generally used in Indian ICUs and empiric choice made for serious ICU related infections. However, this practice may not provide the optimum

Corresponding Author: Suresh Durairajan, Department of Infectious Disease, Apollo Hospitals, Greams Road, Chennai, 600006, India 
outcomes in India, because the prevalence of MDRO varies across and within countries (Kollef and Fraser, 2001; Agarwal et al., 2006; Habibi et al., 2008; Brown et al., 1985). Antibiotic pressure and hence resistant organisms are more likely in the Indian setting. Hence in this study, we sought to explore the epidemiology of infections in Indian ICU patients and determine whether use of western guidelines to initiate empiric antibiotics is appropriate for the Indian setting.

Another factor that has been shown in western studies to improve the quality of antibiotic prescription and de-escalation was consultation by an Infectious Disease (ID) specialist (Byl et al., 1999; Fluckiger et al., 2000). There are no studies that have analyzed the impact of ID consultations on empiric antibiotic selection and de-escalation practices in the Indian ICU setting. Hence, we also evaluated the impact of ID specialist consultations on the appropriateness of initial empiric antibiotic choice and compliance with deescalation practices in our patient population.

\section{MATERIALS AND METHODS}

This prospective observational study was conducted in a 600 bed, tertiary referral hospital in Chennai, India, between January 2009 and July 2009 after obtaining approval from Institutional Review Board for clinical trials. All adult patients (age more than 18 years) admitted to the ICU who had positive culture samples were enrolled. Only data from the first culture of any specimen was used for analysis in patients who had several cultures done. Patients less than 18 years and those with suspected tuberculosis, viral, fungal or parasitic infections were excluded. The isolation of the organism and antimicrobial sensitivity testing were done in accordance with clinical and laboratory standard institute guidelines (Cockerill, 2012). We collected the following data: (1) patient's demographic characteristics; (2) microbiological spectrum and resistance patterns; (3) appropriateness of initial antibiotic choice and de-escalation practices.

\section{RESULTS}

Of the total of 401 patients admitted during the study period $100(25 \%)$ patients had positive cultures. Blood and urine were the commonest samples to become culture positive. $60 \%$ of all cultures grew gram-negative bacilli. Escherichia coli (E.coli) were the commonest gram negative bacilli grown both in blood and urine, whereas Pseudomonas aeruginosa was the most common organism in respiratory secretions.
Table 1. Drug resistant pattern of common pathogens

\begin{tabular}{llr}
\hline Organism & $\begin{array}{l}\text { Resistance } \\
\text { pattern }\end{array}$ & \multicolumn{2}{l}{$\begin{array}{l}\text { Resistance } \\
(\%)\end{array}$} \\
\hline E.coli & Carabapenem Resistance & 5 \\
Pseudomonas aeurginosa & Multidrug Resistance & 35 \\
Acinetobacter baumannii & Multidrug Resistance & 92 \\
\hline
\end{tabular}

Coagulase Negative Staphylococcus (CONS) was the most common gram-positive isolate grown and $9 \%$ of the isolates were Candida.

Forty nine percent of E.coli strains were producing Extended Specturm Beta Lactamas (ESBL positive), while $35 \%$ of P.aeruginosa and $92 \%$ of A. baumanii exhibited Multi Drug Resistance (MDR). Although, the overall incidence of gram positive infections was low, it was noted that about $50 \%$ of the Staphylococcus aureus were Methicillin Resistant (MRSA) (Table 1). Of all patients who had culture positivity $31 \%$ died in the ICU.

The initial empiric choice of antibiotic started was appropriate for 59/122 (48.4\%) organisms. There was no difference in the appropriateness of initial empirical antibiotic choice between patients consulted by an ID specialist and patients where no ID specialist was involved (48.4\% Vs 50.1\%). In 32/59 (54.2\%) organisms de-escalation was possible, but it was done only for $22 / 32(68.7 \%)$ organisms. When ID specialist was involved, appropriate antibiotics were prescribed for $26 / 51$ organisms $(50.1 \%)$ and de-escalation was done for $13 / 14$ organisms $(92.9 \%)$.

\section{DISCUSSION}

Our study is the first large study evaluating the spectrum of microbial flora causing ICU infections and their resistance patterns in India. In this study the prevalence of infections in ICU patients was $25 \%$ $(100 / 401)$. This rate was comparable to previous studies conducted in the developed settings (12-45\%) (Vincent et al., 1995; Richards et al., 1999; Trilla, 1994). Species of gram-negative bacteria such as E.coli, Pseudomonas and Acinetobacter were the most prevalent pathogens $(60 \%)$ recovered from ICU patients in this study; this finding was similar to previous non-ICU studies conducted in India and in ICU studies conducted in other developing countries (Gopalakrishnan and Sureshkumar, 2010; Shehabi and Baadran, 1996; Dai et al., 2006).

The most frequently isolated pathogen in bloodstream and urine was E.coli, while pseudomonas species was the commonest organism in respiratory secretions. In most of the other studies CONS was the 
prevalent bacteria isolated from the bloodstream specimens (Richards et al., 1999). Such a difference might be related to variables such as hand hygiene practices, antibiotic pressure and environmental contamination of the different bacteria in the ICU.

It is well known that multi drug resistant bacteria are becoming increasingly prevalent in ICU environment as result of extensive use of antibiotics. All gram-negative bacteria isolates showed high frequency of resistance to multiple antibiotics but maximum resistance was observed in Acinetobacter isolates (92\%). Concerning resistance patterns of Staphylococcus aureus was observed with $50 \%$ of staphylococcus aureus resistant to methicillin. It was $50 \%$ less compared to studies conducted in Brazil (Toufen et al., 2003).

Our study is also the first study in India to determine the impact of ID specialist consultation on the antimicrobial prescription and de-escalation practices. Although the percentage of patients receiving appropriate antibiotics was not different between patients consulted by an ID specialist and those who were not, de-escalation practices remarkably were higher when ID specialist was involved. It is likely that use of western guidelines for the initial choice led to suboptimal rates of appropriate initial choice of antibiotics in all patients, irrespective of whether an ID specialist was involved or not. It is also likely that high rates of ESBL among E.Coli species and high rates of extreme drug resistance among $P$. aeruginosa and $A$. baumanii (especially respiratory infections) led to lower percentage of patients being covered appropriately by the initial choice of antibiotic. In this study, the overall mortality rate of ICU acquired infections was $31 \%$ and it was comparable to previous studies conducted in other parts of the world where mortality ranged from 10-53.6\% (Ylipalosaari et al., 2006; Goldman and Pier, 1993).

Our study has several limitations. First, our study was conducted in a single tertiary level care ICU and the data may not be applicable across our country or even our region. Second, our ICU is a referral ICU and hence patients often get exposed to antibiotics at other institutions prior to admission here and hence our rates of resistance may be higher than other centers. Third, since we wanted to look at the epidemiology of the bacterial resistance patterns, we did not differentiate colonizers and contaminants from true infections. Fourth, we analyzed only the first cultures of patients, since we looked at initial empiric choice and wanted to select patients at presentation to the ICU, who will likely be antibiotic naïve or minimally exposed to antibiotics. This may have led to falsely low rates of resistance in our study. Last, we did not differentiate between community acquired infections and nosocomial infections, since we wanted to capture the day to day patterns of resistance and antibiotic practices during patients' initial presentation to our ICU.

\section{CONCLUSION}

In conclusion the spectrum of infections in the ICU and the resistance patterns significantly differ from the western world. While gram positive organisms, especially Staphylococcus species predominate in the western world, gram negative organisms are more common in the Indian setting. Furthermore, majority of gram negative infections in the Indian ICU setting have resistance to conventional agents. When choosing empiric antibiotics in acutely ill Indian ICU patients, modifications to western guidelines need to be done using local microbial prevalence and resistance patterns to ensure appropriate antimicrobial coverage. Involvement of an ID specialist seems to increase the rates of antibiotic deescalation. Evolving ICU specific antibiotic pathways and de-escalation policy may improve the overall quality of antibiotic prescription and compliance rates.

\section{REFERENCES}

Agarwal, R., D. Gupta, P. Ray, A.N. Aggarwal and S.K. Jindal, 2006. Epidemiology, risk factors and outcome of nosocomial infections in a Respiratory Intensive Care Unit in North India. J. Infect., 53: 98-105. DOI: 10.1016/j.jinf.2005.10.021

Brown, R.B., D. Hosmer, H.C. Chen, D. Teres and M. Sandas et al., 1985. A comparison of infections in different ICUs within the same hospital. Crit Care Med., 13: 472-476. PMID: 3995999

Byl, B., P. Clevenbergh, F. Jacobs, M.J. Struelens and F. Zech et al., 1999. Impact of infectious diseases specialists and microbiological data on the appropriateness of antimicrobial therapy for bacteremia. Clin. Infect. Dis., 29: 60-66. PMID: 10433566

Cockerill, F.R., 2012. Performance Standards for Antimicrobial Susceptibility Testing. 25th Edn., Clinical and Laboratory Standards Institute, Wayne, PA, ISBN-10: 1562387855, pp: 184.

Dai, C.M., J.D. Zhou, Y. Wu and Y. Qi, 2006. Distribution of pathogen and resistance of nosocomial infections in the intensive care units. Zhong Nan Da Xue Bao Yi Xue Ban, 31: 277-280. PMID: 16706133 
Fluckiger, U., W. Zimmerli, H. Sax, R. Frei and A.F. Widmer, 2000. Clinical impact of an infectious disease service on the management of bloodstream infection. Eur. J. Clin. Microbiol. Infect. Dis., 19: 493-500. PMID: 10968319

Goldman, D.A. and G.B. Pier, 1993. Pathogenesis of infections related to intravascular catheterization. Clin. Microbiol. Rev., 6: 176-192. DOI: 10.1128/CMR.6.2.176

Gopalakrishnan, R. and D. Sureshkumar, 2010. Changing trends in Antimicrobial susceptibility and hospital acquired infections over an 8 year period in a tertiary care hospital in relation to introduction of an infection control programme. J. Assoc. Phys. Ind., 58: 25-31. PMID: 21563610

Habibi, S., N. Wig, S. Agarwal, S.K. Sharma and R. Lodha et al., 2008. Epidemiology of nosocomial infections in medicine intensive care unit at a tertiary care hospital in northern India. Tropical. Doctor, 38: 233-235. DOI: 10.1258/td.2008.070395

Kollef, M.H. and V.J. Fraser, 2001. Antibiotic resistance in the intensive care unit. Ann. Intern. Med., 134: 298-314. PMID: 11182841

Richards, M.J., J.R. Edwards, D.H. Culver and R.P. Gaynes, 1999. Nosocomial infections in medical intensive care units in the United States. National Nosocomial Infections Surveillance System. Crit. Care Med., 27: 887-892. PMID: 10362409
Shehabi, A.A. and L. Baadran, 1996. Microbial infection and antibiotic resistance patterns among Jordanian intensive care patients. East Mediterr Health J., 2: 515-520.

Toufen, J.C., A.L.D. Hovnanian, S.A. Franca and C.R. Carvalho, 2003. Prevalence rates of infection in intensive care units of a tertiary teaching hospital. Rev. Hosp. Clín. Fac. Med. S. Paulo, 58: 254-259. PMID: 14666322

Trilla, A., 1994. Epidemiology of nosocomial infections in adult intensive care units. Intensive Care Med., 20: S1-S4. DOI: 10.1007/BF01745243

Vincent, J.L., D.J. Bihari, P.M. Suter, H.A. Bruining and J. White et al., 1995. The prevalence of nosocomial infection in intensive care units in Europe. Results of the European Prevalence of Infection in Intensive Care (EPIC) Study. EPIC Int. Advisory JAMA, 274: 639-644. PMID: 7637145

Weinstein, R.A., 1998. Nosocomial infection update. Emerg. Infect. Dis., 4: 416-20. PMID: 9716961

Ylipalosaari, P., T.I. Ala-Kokko and J. Laurila, P. Ohtonen and H. Syrjala et al., 2006. Intensive care acquired infection is an independent risk factor for hospital mortality: A prospective cohort study. Crit. Care, 10: R66-R66. DOI: 10.1186/cc4902 